\title{
Heart Rate Variability and Psychometric Analysis in Patients with Hyperactive Heart Fire Syndrome
}

\author{
Oliverio Medina Martínez ${ }^{1}$, Ruben Fossion, 3,4 , Yolanda García Piceno, ${ }^{1,2}$, Rosa E. Lopez-Gomez ${ }^{1,2}$, \\ Emma López-Espinosa ${ }^{1,2}$, Ismael Jiménez-Estrada ${ }^{5}$, Salvador Quiroz-González ${ }^{1,2, *}$ \\ ${ }^{1}$ Department of Acupuncture and Rehabilitation, State University of Ecatepec Valley, Ecatepec State of México, Mexico \\ ${ }^{2}$ Transdiciplinary Health Academic Group (CAST-UNEVE-CA03), Ecatepec State of Mexico, Mexico \\ 3/nstitute for Nuclear Science, National Autonomous University of México, Mexico City, Mexico \\ ${ }^{4}$ Complexity Science Center (C3), National Autonomous University of México, Mexico City, Mexico \\ ${ }^{5}$ Department of Physiology, Biophysics and Neuroscience, Center for Research and Advanced Studies, National Polytechnic Institute, \\ Mexico City, Mexico
}

Received November 3, 2020

Revised June 4, 2021

Accepted June 14, 2021

Correspondence to Salvador Quiroz-González Department of Acupuncture and Rehabilitation, State University of Ecatepec Valley, Ecatepec State of México, Mexico

E-mail sqg20@yahoo.com.mx
Background: Hyperactive heart fire syndrome is characterized by anxiety, insomnia, dream-disturbed sleep, tongue ulcers, heat in the hands, and palpitations. However, syndrome differentiation is often subjective due to a lack of objective, quantifiable variables.

Objectives: To identify changes in heart rate variability (HRV) and psychometric analysis in patients with hyperactive heart fire syndrome.

Methods: Healthy controls $(n=33)$ were compared to patients with hyperactive heart fire syndrome $(n=48)$ from the Integrative University Clinic of the State University of Ecatepec Valley (CIU-UNEVE). Physiological outcome measures included heart rate $(H R)$, the standard deviation of the normal-to-normal heartbeat intervals (SDNN), low (LF) and high frequency (HF) power, and the LF/HF ratio. Psychometric outcome measures included the Athens Insomnia Scale (AIS) and the Hamilton Anxiety Rating Scale (HARS). Results: Compared to controls, hyperactive heart fire patients had higher HR $(9.6 \pm$ $2.62 \%)$, LF (22 $\pm 4.21 \%)$ and LF/HF ratio $(23 \pm 3.14 \%)$, and lower SDNN $(21 \pm 2.33 \%)$ and HF (18 $\pm 4.61 \%)$. Patients showed increased anxiety, both with somatic (33 $\pm 11.2 \%)$ and psychic symptoms (39 $\pm 10.5 \%)$ with more difficulty falling asleep (47 $\pm 9.9 \%)$ and diurnal impact of sleep $(31 \pm 9.6 \%)$.

Conclusion: Hyperactive heart fire patients may have a sympathovagal imbalance due to a reduced parasympathetic tone and/or adominant sympathetic tone, which may be at the origin of the observed symptoms of insomnia and anxiety.

Keywords: Hyperactive heart fire syndrome, Heart rate variability, Anxiety, Insomnia, Palpitations

\section{INTRODUCTION}

Traditional Chinese Medicine (TCM) is an ancient healthcare system, with applicability to several diseases, with both preventive and curative provisions [1-3]. Syndrome differentiation plays a crucial role in TCM to establish diagnoses and decide on therapies, such as moxibustion, acupuncture, herbs, and massage; moreover, it consolidates clinical practice with basic theories of TCM $[2,4]$. Syndrome differentiation is predominantly based on personal experience and interpretation of signs and symptoms by the practitioner $[5,6]$, using usually qualitative (not quantitative) variables $[4,7,8]$, thus constituting a subjective factor in TCM. There is increasing interest in investigating the biomedical origins of these signs and symptoms to provide a more objective interpretation of TCM in line with evidence-based Western medicine and systematize the relationship between specific signs and symptoms and particular syndromes and diseases [8-10].

Within the context of TCM, hyperactive heart fire syndrome is characterized by anxiety, insomnia, dream-disturbed sleep, tongue ulcers, heat in the hands, and palpitations [11]. From a Western perspective, these signs and symptoms may arise in the course of various diseases. However, different syndromes may share a common biomedical basis in TCM [4]. 
The autonomic nervous system (ANS) constitutes a part of the peripheral nervous system that modulates spontaneous physiological processes such as heart rate, arterial pressure, respiration, digestion, and sexual arousal; two of its important divisions are the sympathetic and the parasympathetic system $[12,13]$. Evidence indicates that a maladaptive ANS plays an important role in the pathophysiology of several diseases, including inflammatory bowel disease [14], functional dyspepsia [15], chronic fatigue syndrome [16,17], chronic obstructive pulmonary disease [18], kidney disease [19], cardiovascular disease [20], hepatic diseases [21], type2 diabetes mellitus [22,23], vexation, anxiety, and insomnia $[24,25]$. Heart rate variability (HRV), i.e., the spontaneous variation of heart rate on a beat-to-beat basis, offers a noninvasive and continuous method to quantify ANS activity, and in particular, the contributions of the sympathetic and parasympathetic branches and the sympathovagal balance [26-28], using statistical analysis in the time and/or frequency domain $[22,23]$. HRV is shown to relate to attention regulation, affective information processing, physiological flexibility, cerebral blood flow $[29,30]$, sleep, inflammation, perception, and cognition [31].

In the present study, we hypothesized that the symptoms observed in patients with hyperactive heart fire syndrome, specifically anxiety and sleep impairment, may be related to alterations in the ANS and/or the sympathovagal balance. We compared HRV between controls and hyperactive heart fire patients, applying time-domain analysis such as the standard deviation of normal-to-normal heartbeat intervals (SDNN) and Fourier spectral analysis, including low-frequency power (LF), high-frequency power (HF), and the LF/HF ratio. We also applied questionnaires to assess the level of insomnia and anxiety, using the Athens Insomnia Scale (AIS) and the Hamilton Anxiety Rating Scale (HARS). While focusing on the hyperactive heart fire syndrome using HRV analysis, as a more general objective, we also aim to demonstrate how syndrome differentiation in TCM may be approached, using objective and quantitative methods to unravel the underlying physiopathological mechanisms and their biomedical origins.

\section{MATERIALS AND METHODS}

\section{Ethical aspects}

The research protocol was approved by the Ethics Committee of the State University of Ecatepec Valley (Act. No. 008-2017), following the ethical norms of institutional and national research committees, the Helsinki declaration (and later amendments thereof), and customary international ethical standards. Before the study, all participants were briefed on the purpose and aspects of the research project and signed informed consent.

\section{Study setting}

All study settings were similar to a clinical trial that the authors recently conducted for another TCM syndrome (Olivera-Toro et al. [8]). The present research project was a transverse study conducted from August 2017 to July 2018. A survey was used to acquire data from possible participants in the Integrative University Clinic of the State University of Ecatepec Valley (CIU-UNEVE). A total of 33 volunteers, including 19 (58\%) women and $14(42 \%)$ men, in the age group 45-58 years (average of $50.3 \pm 8.2$ years) and 48 patients with hyperactive heart fire syndrome, including 30 (63\%) women and 18 (38\%) men, in the age group 47-66 years (average of $61.2 \pm 7.6$ years) accepted to participate in the research project. The patient population is a convenience sample of patients with hyperactive heart fire symptoms visiting the university hospital during the research period.

\section{1) Inclusion criteria}

An advertisement was used for enlisting participants from the CIU-UNEVE who met the following requirements: hyperactive heart fire patients according to the Guiding Principles of Clinical Research on Traditional Chinese Medicine [11,32] with the following symptoms:

(1) Primary symptoms directly related to hyperactive heart fire, such as vexation, insomnia, palpitations, and dreamdisturbed sleep.

(2) Secondary symptoms: flushed face, ulceration, and pain in the mouth and/or tongue, rapid pulse.

(3) Diagnostic criteria: A diagnosis of hyperactive heart fire was established in case of a red tongue in combination with 2 primary symptoms or together with 1 primary symptom and at least 2 secondary symptoms.

Two co-authors of the present study, who were licensed acupuncturists, identified TCM syndrome differentiation. A questionnaire classifying different TCM symptoms was developed to check for the inclusion criteria for hyperactive heart fire syndrome. A physical examination was conducted for the tongue and to read the pulse according to the hyperactive heart fire guidelines. Syndrome differentiation was complemented by the Athens Insomnia Scale (AIS) and the Hamilton Anxiety Rating Scale (HARS) to evaluate insomnia and anxiety levels in patients with hyperactive heart fire.

\section{2) Exclusion criteria}

Indications of endocrine, severe psychiatric or psychological disorders, diagnosis of cancer of any nature, inflammatory diseases, such as rheumatoid arthritis, or a clinical history of heart disease were reasons for excluding participants from the study. Any subject on related medication (e.g., psychotropic drugs and caffeine) was excluded. Refusal to sign informed 
consent or incapacity to complete the questionnaires led to exclusion from the study.

\section{Physiological monitoring}

Heart rate variability (HRV) analysis was based on surface electrocardiography (ECG). All recording sessions were conducted at a similar time of the day (from 09:00 to 11:00 AM) to eliminate any possible circadian influence on heart rate. All recordings were made at the same ambient temperature of $25^{\circ} \mathrm{C}$ in a quiet examination room. Measurements were taken in a seated position using an examination chair with knee and trunk-thigh angles of 120 and 135 degrees, respectively.

\section{1) Electrocardiographic (ECG) recordings and heart rate variability (HRV) analysis}

All participants were instructed to rest for $10 \mathrm{~min}$ in a seated position before recording the ECG signal. A detailed description of the measurement equipment, recording procedure, and verification and cleaning of the recorded signals can be found in the study by Olivera-Toro et al. [8]. The derived normal-to-normal (N-N) heartbeat intervals were analyzed in the time and frequency domain using the Kubios HRV software (version 2.2, Matlab Kuopio, Finland) [33]. Measures from the time domain were the average heart rate (HR) and the standard deviation of normal-to-normal heartbeat intervals (SDNN). Indices from the spectral analysis were low-frequency power (LF) or energy of the Fourier power spectrum from 0.04 to $0.15 \mathrm{~Hz}$, high-frequency power (HF), or energy in the power spectrum between 0.15 and $0.40 \mathrm{~Hz}$, where LF is interpreted as an estimate of activity of the sympathetic nervous system, and HF as an estimate of the parasympathetic system and the LF/HF ratio reflects the sympathovagal balance [26-28].

\section{Questionnaires}

\section{1) Anxiety scale}

We used the validated Spanish translation of the Hamilton Anxiety Rating Scale (HARS), which quantifies symptoms related to anxiety and shows good test-retest reliability and concurrent validity [34]. The scale measures psychic and somatic anxiety, using 14 items corresponding to specific symptoms in a range of 0 (absent) to 4 (severe). The total score ranges from $0-56$, where a result of less than 17 corresponds to mild severity, 18-24 with mild to intermediate severity, and 25-30 with intermediate to severe. Cronbach alpha levels for the HARS are 0.92-0.98 [35].

\section{2) Insomnia scale}

We used the validated Spanish translation of the Athens
Insomnia Scale (AIS), which quantifies a variety of insomniarelated symptoms in the form of a short self-report tool, using a total of 8 statements, following the ICD-10 criteria [36]. Each item of the AIS is rated on a scale of 0 (absent) to 3 (severe), the total score ranging from 0-24. The AIS includes sleeprelated symptoms (problems with falling asleep, waking up at night, waking up too early in the morning, sleep duration and quality) and complaints related to daytime functioning (wellbeing, physical and mental acuity, drowsiness). The Cronbach alpha levels for the AIS are above 0.80 [37].

\section{Statistical analysis}

Average values and standard deviation (SD) were calculated for each of the HRV indices mentioned before for each study population and expressed in percent values (\%) for healthy volunteers. Statistical significance was determined using either a parametric Student's t-test or a non-parametric Wilcoxon test, depending on the characteristics of the distribution of the HRV indices. Demographic parameters were compared between both groups using a Student's t-test or a chi-squared test. All statistical tests were performed by blinded biostatisticians using the Graph-Pad Prism software (version 5, San Diego Ca.), and a value of $p<0.05$ was considered statistically significant.

Table 1. Baseline data of healthy controls and patients with hyperactive heart fire

\begin{tabular}{lccc}
\hline \multicolumn{1}{c}{ Characteristic } & $\begin{array}{c}\text { Controls } \\
(\mathrm{n}=33)\end{array}$ & $\begin{array}{c}\text { Hyperactive } \\
\text { heart fire } \\
(\mathrm{n}=48)\end{array}$ & $\begin{array}{c}p> \\
0.05\end{array}$ \\
\hline Demographics & & & \\
Age (years) & $50.3 \pm 8.2$ & $61.2 \pm 7.6$ & $\mathrm{~ns}$ \\
Sex ratio (male/female) & $14 / 19$ & $18 / 30$ & $\mathrm{~ns}$ \\
Weight (kg) & $66.5 \pm 11.2$ & $68.4 \pm 9.7$ & $\mathrm{~ns}$ \\
Height (meters) & $1.8 \pm 0.71$ & $1.6 \pm 0.9$ & $\mathrm{~ns}$ \\
Body mass index (kg/m $\left.{ }^{2}\right)$ & $29 \pm 4.9$ & $27 \pm 5.1$ & $\mathrm{~ns}$ \\
Body fat (\%) & $45 \pm 10.2$ & $38 \pm 14.1$ & $\mathrm{~ns}$ \\
Visceral fat (\%) & $12.1 \pm 1.9$ & $10.3 \pm 4.2$ & $\mathrm{~ns}$ \\
Basal metabolism (Kcal) & $1521 \pm 153$ & $1599 \pm 162$ & $\mathrm{~ns}$ \\
Skeletal muscle (\%) & $27.1 \pm 5.6$ & $28.2 \pm 4.9$ & $\mathrm{~ns}$ \\
Metabolic age (years) & $64 \pm 12.15$ & $65.1 \pm 11.9$ & $\mathrm{~ns}$ \\
Lifestyle factors & & & \\
Smoking & & & \\
No & $30(90.9 \%)$ & $46(95.8 \%)$ & $\mathrm{ns}$ \\
Yes & $3(9.1 \%)$ & $2(4.2 \%)$ & $\mathrm{ns}$ \\
Alcohol consumption & & & \\
No & $27(81.1 \%)$ & $39(82.6 \%)$ & $\mathrm{ns}$ \\
Yes & $6(18.9 \%)$ & $9(17.4 \%)$ & $\mathrm{ns}$ \\
Physical exercise & & & \\
Never or moderate & $31(94.1 \%)$ & $45(93.3 \%)$ & $\mathrm{ns}$ \\
Frequently & $2(5.9 \%)$ & $3(6.7 \%)$ & $\mathrm{ns}$ \\
\hline Ave & & \\
\hline
\end{tabular}

Average values \pm standard deviation are shown. $\mathrm{ns}=$ no significant difference between groups. 


\section{RESULTS}

\section{Participant characteristics}

Table 1 shows the general characteristics of the 2 study groups. A total of 81 participants $(33$ healthy volunteers and 48 patients) were diagnosed with hyperactive heart fire. We detected no significant differences for any of the characteristics included in Table 1.

\section{HRV analysis}

Fig. 1 and Table 2 show the results for HRV indices for the 2 study populations. In the time domain, we found that HR was significantly higher in patients with hyperactive heart fire than in healthy volunteers (panel A), whereas SDNN showed a significant decrease (panel B). In the frequency domain, we found an increase in LF (panel C) and LF/HF (panel E) and a decrease in HF (panel D), and these differences were also significant.

\section{Questionnaires}

\section{1) Anxiety scale}

Fig. 2 and Table 3 show the results for the HARS. Both somatic (panel A) and mental symptoms of anxiety (panel B) were significantly larger in patients with hyperactive heart fire for healthy volunteers.

Table 2. Summary of HRV analysis results of patients with hyperactive heart fire syndrome

\begin{tabular}{lccc} 
Parameter & Controls $(\mathrm{n}=33)$ & $\begin{array}{c}\text { Hyperactive heart } \\
\text { fire }(\mathrm{n}=48)\end{array}$ & $p$ \\
\hline HR & $61 \pm 3.5$ & $68 \pm 4.2^{*}$ & 0.016 \\
SDNN (ms) & $63 \pm 9.4$ & $49 \pm 6.6^{* *}$ & 0.001 \\
LF & $808 \pm 103$ & $1129 \pm 95.9^{* *}$ & 0.003 \\
HF & $1020 \pm 127$ & $867 \pm 91.5^{* *}$ & 0.001 \\
LF/HF & $1.1 \pm 0.2$ & $1.4 \pm 0.4^{* *}$ & 0.002 \\
\hline
\end{tabular}

Mean values $( \pm S E)$ for the parameter of each group. HRV = Heart Rate Variability.

${ }^{*} p<0.05$ vs. controls. ${ }^{* *} p<0.01$ vs. controls.
A
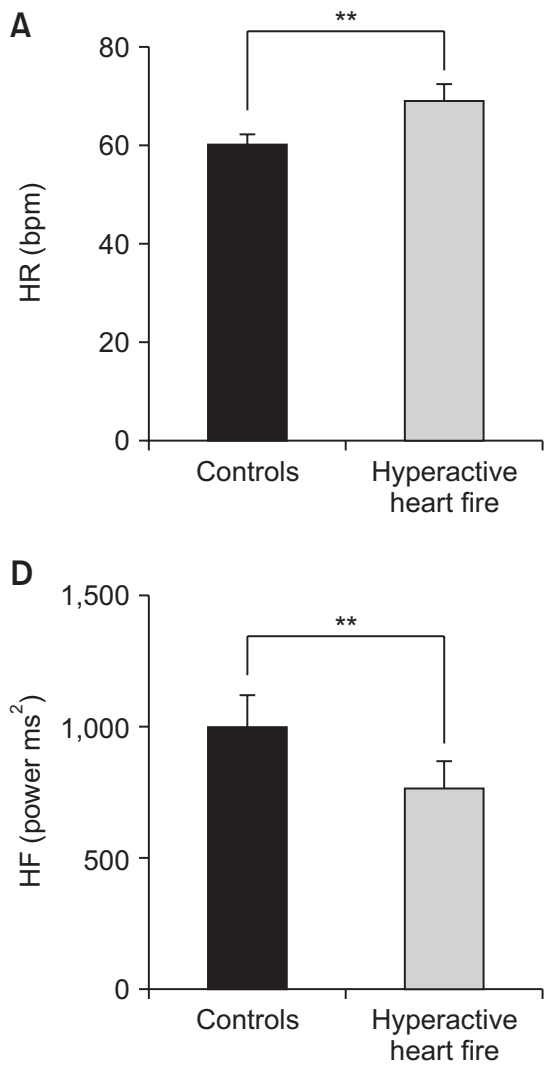

B

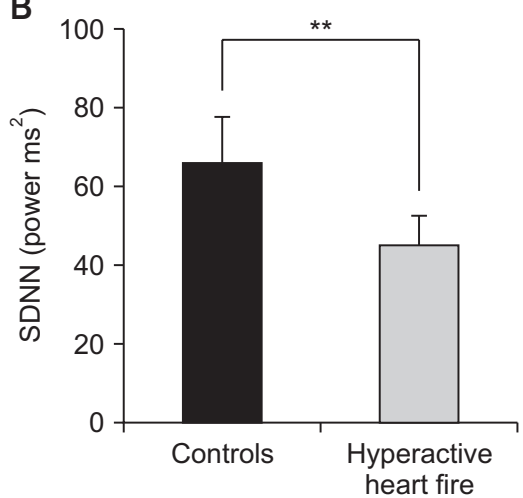

E

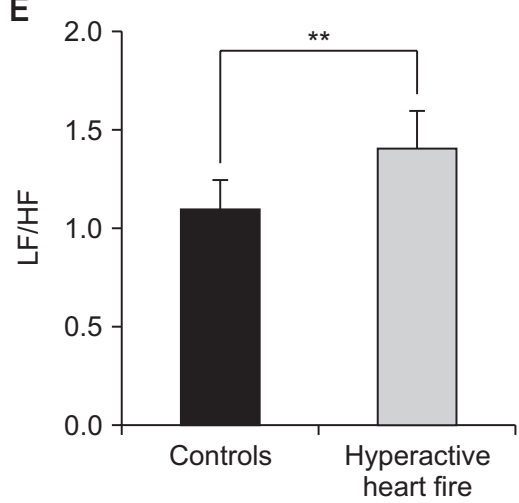

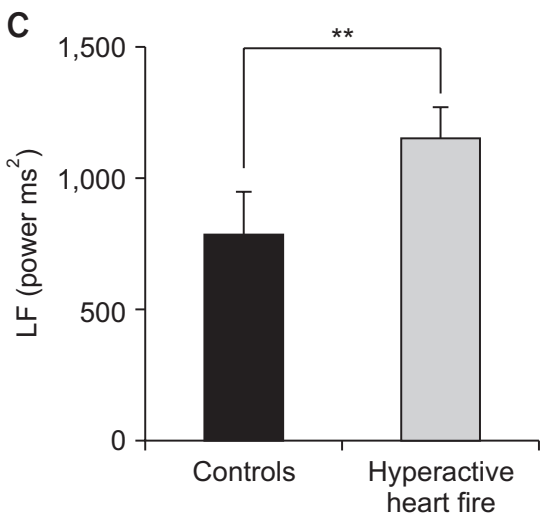

Fig. 1. Average heart rate (HR) and heart rate variability $(H R V)$ parameters in healthy volunteers $(n=33)$ and patients with hyperactive heart fire syndrome $(n=48)$ are shown as average values \pm standard deviation (SD). (A) Average heart rate (HR). (B) Standard deviation of normalto-normal heartbeat intervals (SDNN). (C) Low-frequency power (LF). (D) High-frequency power (HF). (E) LF/HF ratio. Bars indicate $* * p<0.01$, $* p<0.05$. 


\section{2) Insomnia scale}

Fig. 3 and Table 3 show the results for the AIS. Both sleeprelated symptoms (panel A) and complaints related to daytime functioning (panel B) were significantly higher in patients with hyperactive heart fire for healthy volunteers.

\section{DISCUSSION}

\section{Autonomous nervous system and heart rate variability (HRV)}

The present study used HRV as a non-invasive physiological measure to evaluate (for the first time) the state of the autonomous nervous system in patients with hyperactive heart fire. The patients showed a significantly larger HR and LF and a significantly lower SDNN, thus indicating an increased dominance of the sympathetic nervous system in healthy volunteers. However, HF showed a significant decrease, indicating a less active parasympathetic nervous system. The LF/HF ratio was significantly higher in patients than in healthy volunteers, suggesting an increased sympathetic and/or decreased parasympathetic activity. Therefore, it seems reasonable to infer that hyperactive heart fire syndrome is related to an altered sympathovagal balance.

\section{Anxiety}

Anxiety disorders are among the most prevalent and the most costly psychiatric conditions [38]. Anxiety can be interpreted as a reduced capacity to inhibit cognitive (e.g., apprehension, vigilance, and worry), affective (e.g., panic), behavioral (e.g., avoidance), and physiological (e.g., increased $\mathrm{HR}$ ) responses [39]. Anxiety disorders are also related to reduced vagal outflow and decreased HRV [39]. In the present study, patients with hyperactive heart fire showed

Table 3. Summary of psychometric analysis results of patients with hyperactive heart fire syndrome

\begin{tabular}{lccc}
\hline \multicolumn{1}{c}{ Scale } & $\begin{array}{c}\text { Controls } \\
(\mathrm{n}=33)\end{array}$ & $\begin{array}{c}\text { Hyperactive } \\
\text { heart fire } \\
(\mathrm{n}=48)\end{array}$ & $p$ \\
\hline $\begin{array}{l}\text { Hamilton anxiety rating scale } \\
\text { Psychic symptom }\end{array}$ & $100 \pm 8.2 \%$ & $133 \pm 11.2 \% *$ & 0.024 \\
$\begin{array}{l}\text { Somatic symptoms } \\
\text { Athens insomnia scale } \\
\quad \text { Difficulty of nighttime } \\
\text { sleep }\end{array}$ & $100 \pm 9.1 \%$ & $139 \pm 10.5 \% \%^{* *}$ & 0.001 \\
\hline Daytime impact & $100 \pm 7.9 \%$ & $147 \pm 9.9 \% * *$ & 0.001 \\
\hline
\end{tabular}

Normalized values indicating the average and standard deviation (SD) expressed as a percentage (\%) for healthy volunteers. ${ }^{*} p<0.05$ vs. controls. ${ }^{* *} p<0.01$ vs. controls.
A

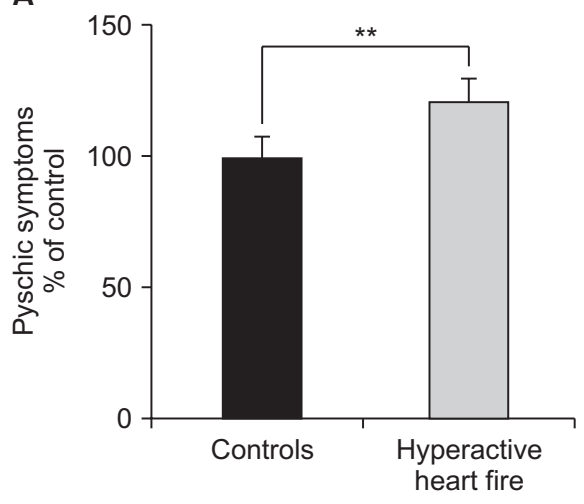

A

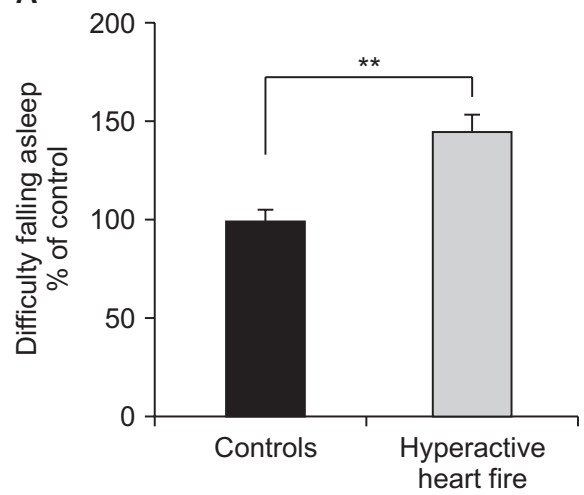

B

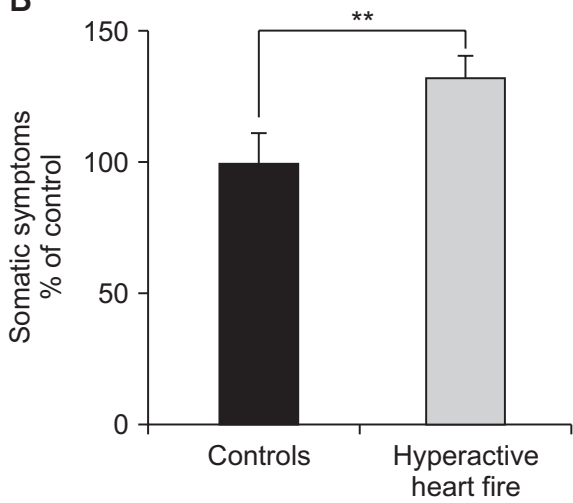

B

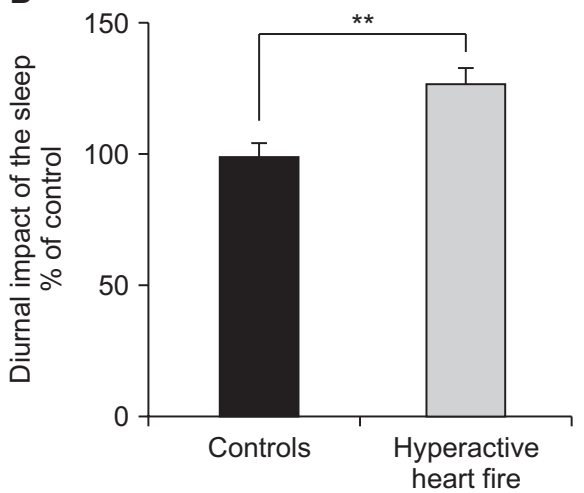

Fig. 2. Hamilton Anxiety Rating Scale (HARS) in healthy volunteers ( $\mathrm{n}$ = 33) and patients with hyperactive heart fire syndrome $(n=48)$. (A) Psychic symptom, (B) Somatic symptoms. Shown are normalized values indicating the average and standard deviation (SD) expressed as a percentage $(\%)$ for healthy volunteers. Bars indicate ${ }^{* *} p<0.01$.

Fig. 3. Athens Insomnia Scale (AIS) in healthy volunteers $(n=33)$ and the patient group with hyperactive heart fire $(n=48)$. (A) Difficulty of nighttime sleep, (B) daytime impact. Shown are normalized values indicating the average and standard deviation (SD) expressed as a percentage (\%) for healthy volunteers. Bars indicate ${ }^{* *} p<0.01$. 
a significant increase in anxiety levels in combination with a higher HR and a lower HRV (reduced SDNN) for healthy volunteers.

\section{Insomnia}

Insomnia is the most frequent sleep complaint, with $9-15 \%$ of the world population affected by nighttime symptoms and their daytime consequences [24]. Various aspects of sleep are related to the functioning of the autonomic nervous system, including subjective sleep quality, sleep latency, and the use of sleep medication $[24,25]$. Specifically, studies show that decreased sleep quality corresponds to increased sympathetic activity and lower HRV, although it is unclear whether problems with sleep are the cause or the result of increased sympathetic activation [25]. In the present study, for healthy volunteers, we observed that hyperactive heart fire patients showed significantly more alterations in nighttime sleep and daytime consequences in combination with a reduced HRV (lower SDNN) and evidence for an increased sympathetic tone and/or a decreased parasympathetic tone.

\section{HRV as a biological marker in hyperactive heart fire and other TCM syndromes}

In Traditional Chinese Medicine (TCM), a syndrome may imply both physical and mental symptoms. The pathophysiologic alterations in hyperactive heart fire are associated with fire hyperactivity and impairment of heart yang qi and its heating and driving functions, including blood circulation and "Shen," usually translated as "spirit" or "mind." Such hyperactivity produces anxiety, insomnia, palpitations, dream-disturbed sleep, tongue ulcers, and heat in the hands [11]. From the perspective of Western medicine, heart rate variability (HRV) may also be relevant for physical and psychological well-being. Studies show that HRV is reduced in diabetes $[22,23]$ and obesity [30], concentration problems, emotional dysregulation [40], depression [41], anxiety, and sleep impairment [24,25].

Evidence shows that a common denominator may exist in the pathogenesis of specific diseases. ANS impairment may be a common denominator that decreases the capacity for adaptation to perturbations and challenges from the external environment and the maintenance of a stable internal environment, as evidenced by HRV [29,30] and variability of other important physiological variables, such as blood pressure, body temperature, and physical activity [42-45].

In a recent publication, we found evidence for an altered ANS in another TCM syndrome, the Spleen-Qi Deficiency Syndrome (SQDS), with a similar higher HR, reduced HRV (lower SDNN), increased LF and LF/HF, and a lower HF, possibly due to an increased sympathetic tone and/ or decreased parasympathetic tone, and also producing alterations in intestinal peristalsis, gastrointestinal symptoms, fatigue, with a decrease in attention [8].

At first sight, it may seem counterintuitive that we found similar results for HRV in a hyperactivity and deficiency syndrome, in particular for SDNN, LF, HF, and LF/HF. However, from a control theory perspective, the heart is an effector organ, and heart rate plays the role of a "regulating variable" to maintain the corresponding "regulated variable" of blood pressure as close as possible to its set point of 120/80 $\mathrm{mmHg}$ [46]. The function of heart rate variability is to adapt to or absorb external and internal perturbations and maintain constant blood pressure. Therefore, HRV may be higher and blood pressure variability (BPV) lower in health. However, in adverse conditions of aging and/or disease, HRV is decreased (loss of adaptive capacity), and consequently, BPV may increase (loss of Claude Bernard's constant "milieu intérieur" or internal environment) [43]. Therefore, global HRV measures such as SDNN may decrease in hyperactivity and deficiency syndromes.

In the decomposition of HRV variability in LF and HF, the majority of adverse health conditions are associated with increased LF, decreased HF, and/or increased LF/ $\mathrm{HF}$, including various cardiovascular pathologies, such as hypertension, congestive heart failure, mitral valve prolapse, and cardiomyopathy [26], diabetes [47], fibromyalgia [48], chronic fatigue [49], both hyper and hypothyroidism [50], aging [51], and stress, panic, anxiety, or worry [27]. Reports show that only a few diseases are correlated with a decreased LF and increased HF and/or decreased LF/HF, such as scleroderma [52] and asthma [53]. This finding may be explained as follows. Intrinsic heart rate, i.e., heart rate without autonomic modulation, is about $100 \mathrm{bpm}$ in the healthy adult, higher in newborns, and decreases with age. The parasympathetic nervous system is active in "rest and digest" conditions, lowering the average heart rate to about $60 \mathrm{bpm}$. Vagolysis or parasympathetic inhibition is the first adaptive response to a stressor (e.g., standing up from a seated or lying position) that increases heart rate to compensate for the drop in blood pressure due to orthostatic challenge. It has the advantage of being an immediate response and does not cost any additional energy because it corresponds to "switching off" that particular branch of the autonomous nervous system. Apart from inhibiting the parasympathetic nervous system, the "fight and fight" challenge involves sympathetic nervous system activation, increasing heart rate to values above $100 \mathrm{bpm}$. The dynamic range of heart rate, i.e., the minimum and maximum heart rate achievable by parasympathetic nervous system or sympathetic nervous system activation, decreases with age and is aggravated by sedentarism and suboptimal physical conditions (Fig. 4). In these cases, vagolysis may not offer a sufficient first- 


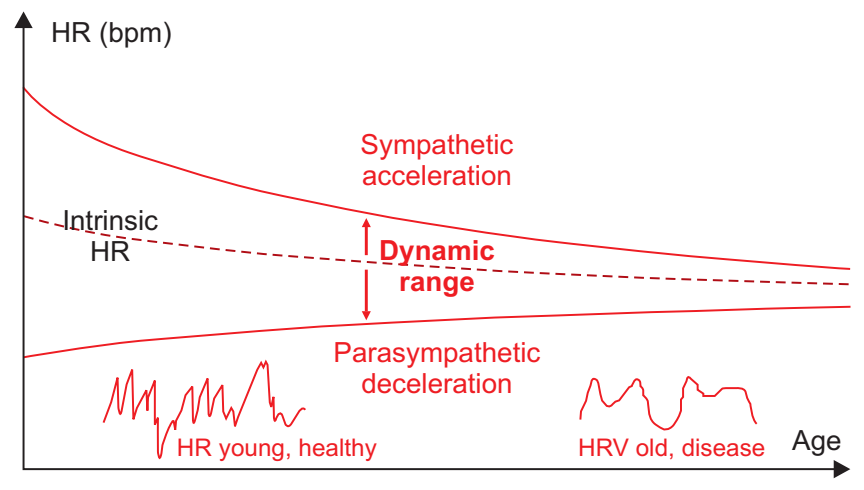

Fig. 4. Average heart rate $(\mathrm{HR})$ and heart rate variability $(\mathrm{HRV})$ as a function of age.

line response to stressors, and sympathetic nervous system activation may compensate for minimal physical efforts such as standing up or walking, corresponding to an increased average heart rate, a decrease in SDNN and HF, and an increase in LF and LF/HF. We assume that something similar will be observed in most chronic-degenerative diseases imposing chronic stress and seems to be the case for TCM deficiency and excess syndromes discussed here. Contrarily, few diseases seem to result from the incapacity of the parasympathetic nervous system to decrease its activity (e.g., asthma with excessive constriction of the bronchioles). Cytokine production by the immune system plays an important role in pathophysiological processes. Increased expression of pro-inflammatory cytokines can lead to tissue injury and damage, as is evident in chronic inflammatory diseases, such as inflammatory bowel disease, rheumatoid arthritis, and atherosclerosis [54]. Furthermore, subclinical inflammation, such as low-grade systemic inflammation, is associated with diabetes, gut dysbiosis, colitis, gastrointestinal impairment, cardiovascular disease, obesity, anxiety, insomnia, and psychiatric disorders [54,55], such that inflammatory markers are significant predictors of mortality in older adults [56].

Several clinical or preclinical studies have shown that HRV is inversely correlated with biomarkers of systemic inflammation and subclinical inflammation in healthy individuals and those with diseases [57,58]. Specifically, decreased HRV correlates with elevations in circulating C-reactive proteins (CRP), interleukin (IL)-6, and fibrinogen in healthy individuals [57,58]. Moreover, increased heart rate and reduced heart rate variability are associated with subclinical inflammation in healthy middle-aged and elderly subjects [59]. It has also been proposed that HRV may be (in some cases) a potential predictor for subsequent problems and/or an index of disease progression.

Evidence shows that vagal tone is central to regulating several allostatic systems, such as the cardiovascular system, glucose regulation, the hypothalamic-pituitary-adrenal axis function, and inflammatory processes $[60,61]$. The central nervous system can decrease cytokine production via vagal nerve activity $[62,63]$. Therefore, it is biologically plausible that an altered autonomic balance can trigger low-grade inflammation, gradually leading to loss of adaptive capacity and symptoms associated with hyperactive heart fire as palpitations, anxiety, and insomnia.

The brain can affect the community structure and function of the gut microbiota through the vagus nerve of the autonomous nervous system by modulating regional gut motility, intestinal transit and secretion, gut permeability, and potentially through the luminal secretion of hormones that directly modulate microbial gene expression. Consequently, decreased vagus nerve activity may also be associated with spleen-deficiency syndrome symptoms, such as gastrointestinal impairment, immune alterations, fatigue, poor memory, and reduced concentration [8].

Furthermore, factors associated with spleen qi deficiency syndrome and low-grade inflammation correlated with a poor diet also constitute risk factors for dysbiosis, leading to anxiety and insomnia through bidirectional interactions within the gut-microbiome-immune-brain axis. Moreover, mental stress also leads to gastrointestinal alterations via the brain-gut-microbiome axis [64]. Thus, different deficiency and excess syndromes in TCM may be characterized by common denominators, such as low HRV and similar impairment of the sympathetic-vagal balance, although it is unknown what originates first (Fig. 5). It is feasible that lowgrade systemic inflammation, as evidenced by low HRV, also contributes to deficiency or excess syndromes in TCM, but further studies are necessary to test this hypothesis.

\section{Relationship between meridians, acupuncture treatment, and heart rate variability}

As previously shown, HRV is a non-invasive physiological measure to evaluate the state of the autonomous nervous system. The present study on hyperactive heart fire syndrome and our previous contribution on the spleen-qi deficiency syndrome [8] discussed the role of HRV as an objective and quantitative tool for syndrome differentiation. Most researchers agree that acupuncture modulates the autonomic nervous system. Accumulating evidence shows that HRV may also be used to evaluate the effects of acupuncture (Fig. 6) in health and pathological conditions [65].

After electroacupuncture stimulation at the Taichong (LR3) acupoint, heart rate slowed down, and the spectral power density of HRV increased [66]. Acupuncture at the bladder meridian [Dachangshu (BL25), Zhibian (BL54)], gallbladder meridian [Huantiao (GB30), Fengshi (GB31), Yanglingquan (GB34), Xuanzhong (GB39)], and liver meridian [Taichong 


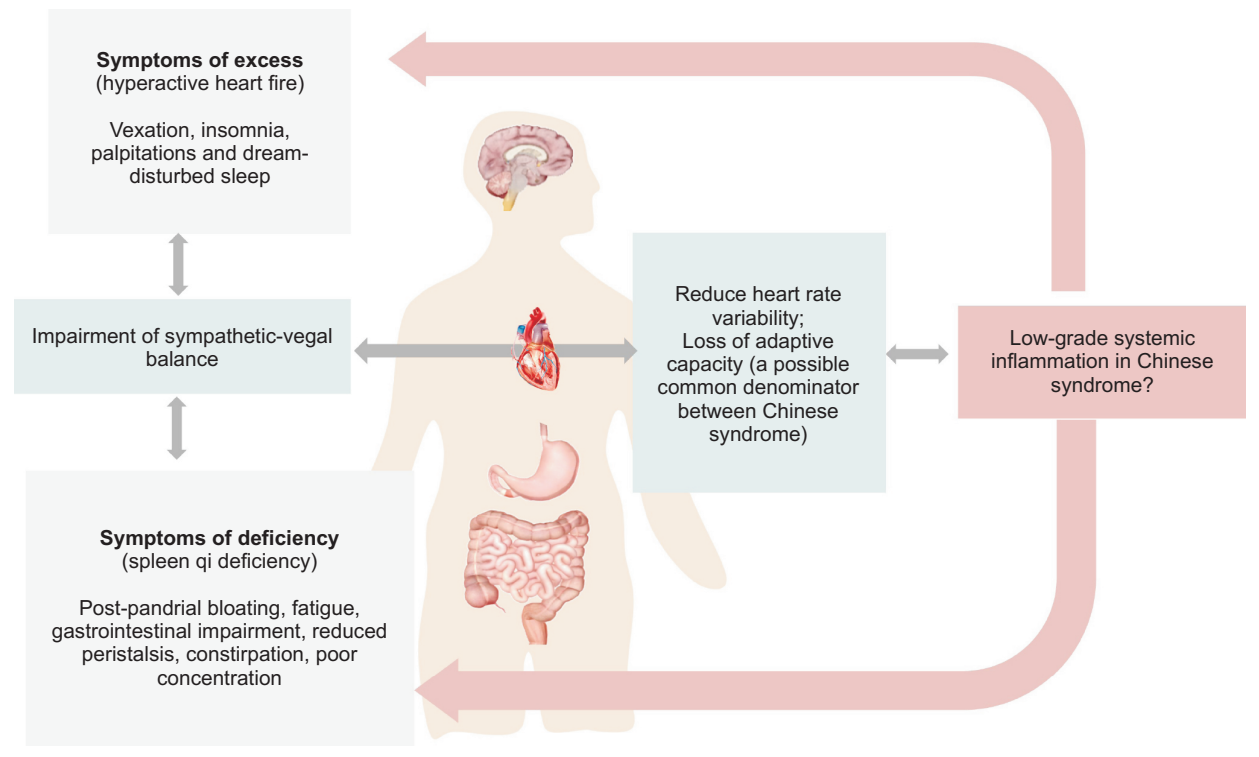

Fig. 5. Hypothetical explanation of the changes in the heart rate variability (HRV) and sympatheticvagal balance in hyperactive heart fire syndrome and spleen qi deficiency syndrome.

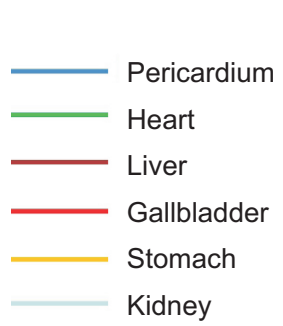

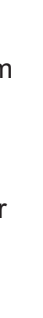
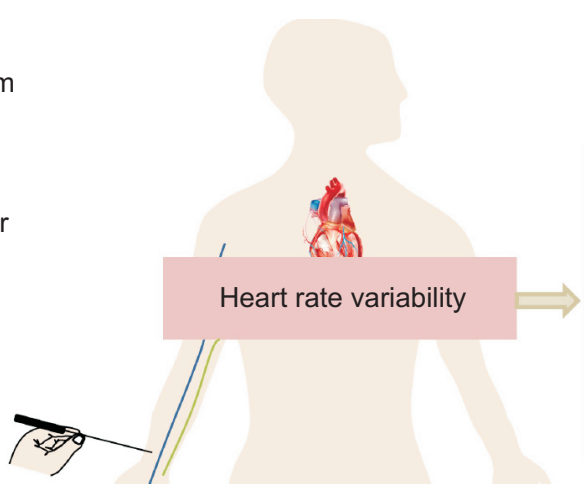

Increase heart rate variability; Improvement sympathovagal balance;

Recovery of adaptive capacity through summative effect of acupuncture stimulation
Fig. 6. Summary of some acupuncture meridians that show effects on heart rate variability (HRV). It is proposed that a summative effect by the stimulation of acupuncture points produced recovery of adaptive capacity of the organism through the modulation of the sympathetic-vagal balance.
(LR3)] improve the balance of the ANS in patients during lumbosacral acupuncture treatment [67]. Additional research has evaluated the effect on HRV parameters of needling at specific acupoints located at the pericardium meridian [Neiguan (PC6)] [68,69], heart meridian [Sobu (HT8)] [70], bladder meridian [Shenmai (BL62)], kidney meridian [Zhaohai (KI6)] [71], and liver meridian [72].

Researchers propose that some acupoints and meridians may have stronger influences on HRV than others. In this way, Sung [73] investigated the effect of stimulation of acupoints at different meridians along the arm and leg on the heart rate. The acupoint Quze (PC3), located in the pericardium meridian along the arm, increased SDNN, but there was little change at acupoint Ququan (LR8) [73]. Also, Shaofu (HT8), an acupoint located in the heart meridian along the arm, decreased sympathetic activity and increased parasympathetic nervous activity [70].

Another study found significant changes in HRV parameters in relaxed and stressed conditions after stimulation of acupoints located in arms [Shenmen (HT7), Neiguan 
PC6, Hegu (LI4)] and legs [Taichong (LR3), Zusanli (ST36)] [74]. SDNN was significantly increased after acupuncture stimulation at normal times, whereas LF/HF ratio and LFnorm were significantly decreased, and HFnorm showed a significant increase after acupuncture stimulation at a term of examination stress in students [74]. Studies showed that an acupoint located in the stomach meridian at the lower extremities, Zusanli (ST36), enhanced the gastric myoelectrical activity and accelerated gastric emptying through the vagal pathway [75]. Acupuncture applied to Shenmai (BL62) induced significant changes in SDNN, while stimulation of Zhaohai (KI6) and sham acupuncture applied to non-acupoints did not induce any significant changes in SDNN [76].

Other studies showed that acupuncture applied during resting states in healthy subjects reduced vagal tone or did not modify the sympathovagal balance. Acupuncture attenuated sympathoexcitation under stress or in subjects with abnormal sympathoexcitation $[77,78]$.

Thus, the specificity of acupoints and meridians in HRV remains unclear due to multiple factors such as single or combination acupuncture stimulation, the number of rotations, the acupuncture point as a dynamic process, physiological states, including negative expectations such as anxiety and stress states generated during the acupuncture intervention, including "DeQi" responses $[71,79,80]$. Therefore, further studies are necessary to clarify the specificity of acupoints and meridians in HRV.

\section{CONCLUSIONS}

Hyperactive heart fire syndrome may be related to a sympathovagal imbalance with low vagal tone and/or increased sympathetic tone, producing anxiety and insomnia. HRV may be used as a biomarker in syndrome differentiation of hyperactive heart fire and other TCM syndromes.

\section{ACKNOWLEDGEMENTS}

Jiménez-Estrada, S. Quíroz-González, and R. Fossion acknowledge partial support from fellowships from the Sistema Nacional de Investigadores (SNI) from CONACyT. R. Fossion is grateful for financial support from the Universidad Nacional Autónoma de México through DGAPA with grant PAPIIT IN110321.

\section{CONFLICT OF INTEREST}

The authors declare no conflict of interest.

\section{ORCID}

Oliverio Medina Martínez, https://orcid.org/0000-0002-6828-1094

Ruben Fossion, https://orcid.org/0000-0001-8456-2075 Yolanda García Piceno,

Rosa E. Lopez-Gomez, https://orcid.org/0000-0002-5342-2771

Emma López-Espinosa, https://orcid.org/0000-0001-5315-4618

Ismael Jiménez-Estrada, https://orcid.org/0000-0002-4832-5328

Salvador Quiroz-González, https://orcid.org/0000-0003-2751-3554 https://orcid.org/0000-0002-0100-5060

\section{REFERENCES}

1. Chen YB, Tong XF, Ren J, Yu CQ, Cui YL. Current research trends in traditional Chinese medicine formula: a bibliometric review from 2000 to 2016. Evid Based Complement Alternat Med 2019;2019:3961395.

2. Jiang M, Lu C, Zhang C, Yang J, Tan Y, Lu A, et al. Syndrome differentiation in modern research of traditional Chinese medicine. J Ethnopharmacol 2012;140:634-42.

3. Quiroz-González S, Olivera Toro A, García Piceno Y, JiménezEstrada I, Fossion R. Psychoneurobiomodulation: an emerging concept to understand the systemic effects of neurophysiological acupuncture. Longhua Chin Med 2019;2:21.

4. Cheng F, Wang X, Song W, Lu Y, Li X, Zhang H, et al. Biologic basis of TCM syndromes and the standardization of syndrome classification. J Tradit Chin Med Sci 2014;1:92-7.

5. Mist S, Ritenbaugh C, Aickin M. Effects of questionnairebased diagnosis and training on inter-rater reliability among practitioners of traditional Chinese medicine. J Altern Complement Med 2009;15:703-9.

6. Zhang GG, Singh B, Lee W, Handwerger B, Lao L, Berman B. Improvement of agreement in TCM diagnosis among TCM practitioners for persons with the conventional diagnosis of rheumatoid arthritis: effect of training. J Altern Complement Med 2008;14:381-6.

7. Chen L, Yang Z, Chen W, Li R, Lin C, Guan L, et al. Differential expression of immune-related genes between healthy volunteers and type 2 diabetic patients with spleen-deficiency pattern. J Tradit Chin Med 2015;35:646-52.

8. Olivera-Toro A, Fossion R, Li L, López-Gómez RE, LópezEspinosa E, Jiménez-Estrada $\mathrm{I}$, et al. Changes in heart rate variability in patients with spleen-qi deficiency syndrome. J Acupunct Meridian Stud 2019;12:111-21.

9. Yoo J, Lee E, Kim C, Lee J, Lixing L. Sasang constitutional medicine and traditional Chinese medicine: a comparative 
overview. Evid Based Complement Alternat Med 2012;2012: 980807.

10. Zheng S, Kim C, Meier P, Sibbritt D, Zaslawski C. Development of a novel questionnaire for the traditional Chinese medicine pattern diagnosis of stress. J Acupunct Meridian Stud 2017;10: 276-85.

11. Dong J, Wang T, Zhao L, Chen X. Pattern of disharmony between the heart and kidney: theoretical basis, identification and treatment. J Tradit Chin Med Sci 2017;4:317-21.

12. Waxenbaum JA, Reddy V, Varacallo M. Anatomy, autonomic nervous system. [Updated 2019 Mar 9]. In: StatPearls [Internet]. Treasure Island (FL): StatPearls Publishing; 2020. Available from: https://www.ncbi.nlm.nih.gov/books/NBK539845/

13. Meisel C, Schwab JM, Prass K, Meisel A, Dirnagl U. Central nervous system injury-induced immune deficiency syndrome. Nat Rev Neurosci 2005;6:775-86.

14. Taylor CT, Keely SJ. The autonomic nervous system and inflammatory bowel disease. Auton Neurosci 2007;133:104-14.

15. Tominaga K, Fujikawa Y, Tsumoto C, Kadouchi K, Tanaka F, Kamata N, et al. Disorder of autonomic nervous system and its vulnerability to external stimulation in functional dyspepsia. J Clin Biochem Nutr 2016;58:161-5.

16. Orjatsalo M, Alakuijala A, Partinen M. Autonomic nervous system functioning related to nocturnal sleep in patients with chronic fatigue syndrome compared to tired controls. J Clin Sleep Med 2018;14:163-71.

17. Cvejic E, Sandler CX, Keech A, Barry BK, Lloyd AR, VollmerConna U. Autonomic nervous system function, activity patterns, and sleep after physical or cognitive challenge in people with chronic fatigue syndrome. J Psychosom Res 2017; 103:91-4.

18. Mohammed J, Meeus M, Derom E, Da Silva H, Calders P. Evidence for autonomic function and its influencing factors in subjects with COPD: a systematic review. Respir Care 2015;60: 1841-51.

19. Hering D, Esler MD, Schlaich MP. Chronic kidney disease: role of sympathetic nervous system activation and potential benefits of renal denervation. EuroIntervention 2013;9 Suppl R:R127-35.

20. La Rovere MT, Christensen JH. The autonomic nervous system and cardiovascular disease: role of n-3 PUFAs. Vascul Pharmacol 2015;71:1-10.

21. Hajiasgharzadeh K, Baradaran B. Cholinergic anti-inflammatory pathway and the liver. Adv Pharm Bull 2017;7:507-13.

22. Rivera AL, Estañol B, Sentíes-Madrid H, Fossion R, ToledoRoy JC, Mendoza-Temis J, et al. Heart rate and systolic blood pressure variability in the time domain in patients with recent and long-standing diabetes mellitus. PLoS One 2016;11: e0148378.

23. Rivera AL, Estañol B, Fossion R, Toledo-Roy JC, CallejasRojas JA, Gien-López JA, et al. Loss of breathing modulation of heart rate variability in patients with recent and long standing diabetes mellitus Type II. PLoS One 2016;11:e0165904.

24. Ohayon MM. Epidemiology of insomnia: what we know and what we still need to learn. Sleep Med Rev 2002;6:97-111.

25. Oliver MD, Baldwin DR, Datta S. The relationship between sleep and autonomic health. J Am Coll Health 2020;68:550-6.

26. Heart rate variability: standards of measurement, physiological interpretation and clinical use. Task Force of the European Society of Cardiology and the North American Society of Pacing and Electrophysiology. Circulation 1996;93:1043-65.

27. Shaffer F, McCraty R, Zerr CL. A healthy heart is not a metronome: an integrative review of the heart's anatomy and heart rate variability. Front Psychol 2014;5:1040.

28. Shaffer F, Ginsberg JP. An overview of heart rate variability metrics and norms. Front Public Health 2017;5:258.

29. Thayer JF, Lane RD. A model of neurovisceral integration in emotion regulation and dysregulation. J Affect Disord 2000;61: 201-16.

30. Thayer JF, Lane RD. Claude Bernard and the heart-brain connection: further elaboration of a model of neurovisceral integration. Neurosci Biobehav Rev 2009;33:81-8.

31. Bonaz B, Sinniger V, Pellissier S. Vagal tone: effects on sensitivity, motility, and inflammation. Neurogastroenterol Motil 2016;28:455-62.

32. Zheng XY. Guiding principles of clinical research on traditional Chinese medicine. Beijing: China Medical Science and Technology Press, 2002.

33. Tarvainen MP, Niskanen JP, Lipponen JA, Ranta-Aho PO, Karjalainen PA. Kubios HRV--heart rate variability analysis software. Comput Methods Programs Biomed 2014;113:210-20.

34. Porter E, Chambless DL, McCarthy KS, DeRubeis RJ, Sharpless BA, Barrett MS, et al. Psychometric properties of the reconstructed Hamilton depression and anxiety scales. J Nerv Ment Dis 2017;205:656-64.

35. Lobo A, Chamorro L, Luque A, Dal-Ré R, Badia X, Baró E. [Validation of the Spanish versions of the Montgomery-Asberg depression and Hamilton anxiety rating scales]. Med Clin (Barc) 2002;118:493-9. Spanish.

36. Soldatos CR, Dikeos DG, Paparrigopoulos TJ. Athens Insomnia Scale: validation of an instrument based on ICD-10 criteria. J Psychosom Res 2000;48:555-60.

37. Gómez-Benito J, Ruiz C, Guilera G. A Spanish version of the Athens Insomnia Scale. Qual Life Res 2011;20:931-7.

38. Kessler RC, Berglund P, Demler O, Jin R, Merikangas KR, Walters EE. Lifetime prevalence and age-of-onset distributions of DSM-IV disorders in the National Comorbidity Survey Replication. Arch Gen Psychiatry 2005;62:593-602.

39. Chalmers JA, Quintana DS, Abbott MJ, Kemp AH. Anxiety disorders are associated with reduced heart rate variability: a meta-analysis. Front Psychiatry 2014;5:80.

40. Friedman BH, Thayer JF. Anxiety and autonomic flexibility: a cardiovascular approach. Biol Psychol 1998;49:303-23. 
41. Thayer JF, Smith M, Rossy LA, Sollers JJ, Friedman BH. Heart period variability and depressive symptoms: gender differences. Biol Psychiatry 1998;44:304-6.

42. Fossion R, Rivera AL, Toledo-Roy JC, Ellis J, Angelova M. Multiscale adaptive analysis of circadian rhythms and intradaily variability: application to actigraphy time series in acute insomnia subjects. PLoS One 2017;12:e0181762.

43. Fossion R, Rivera AL, Estañol B. A physicist's view of homeostasis: how time series of continuous monitoring reflect the function of physiological variables in regulatory mechanisms. Physiol Meas 2018;39:084007.

44. Fossion R, Fossion JPJ, Rivera AL, Lecona OA, Toledo-Roy JC, García-Pelagio KP, et al. Homeostasis from a time-series perspective: an intuitive interpretation of the variability of physiological variables. In: Olivares-Quiroz L, Resendis-Antonio O, eds. Quantitative Models for Microscopic to Macroscopic Biological Macromolecules and Tissues. Cham: Springer, 2018: 87-109.

45. Fossion R, Sáenz-Burrola A, Zapata-Fonseca L. On the stability and adaptability of human physiology: Gaussians meet heavytailed distributions. Interdisciplina 2020;8:55-81.

46. Modell H, Cliff W, Michael J, McFarland J, Wenderoth MP, Wright A. A physiologist's view of homeostasis. Adv Physiol Educ 2015;39:259-66.

47. Manzella D, Paolisso G. Cardiac autonomic activity and Type II diabetes mellitus. Clin Sci (Lond) 2005;108:93-9.

48. Cohen H, Neumann L, Shore M, Amir M, Cassuto Y, Buskila D. Autonomic dysfunction in patients with fibromyalgia: application of power spectral analysis of heart rate variability. Semin Arthritis Rheum 2000;29:217-27.

49. Escorihuela RM, Capdevila L, Castro JR, Zaragozà MC, Maurel $\mathrm{S}$, Alegre J, et al. Reduced heart rate variability predicts fatigue severity in individuals with chronic fatigue syndrome/myalgic encephalomyelitis. J Transl Med 2020;18:4.

50. Chen JL, Chiu HW, Tseng YJ, Chu WC. Hyperthyroidism is characterized by both increased sympathetic and decreased vagal modulation of heart rate: evidence from spectral analysis of heart rate variability. Clin Endocrinol (Oxf) 2006;64:611-6.

51. Rastović M, Srdić-Galić B, Barak O, Stokić E, Polovina S. Aging, heart rate variability and metabolic impact of obesity. Acta Clin Croat 2019;58:430-8.

52. Sallam H, McNearney TA, Doshi D, Chen JD. Transcutaneous electrical nerve stimulation (TENS) improves upper GI symptoms and balances the sympathovagal activity in scleroderma patients. Dig Dis Sci 2007;52:1329-37.

53. Lutfi MF. Autonomic modulations in patients with bronchial asthma based on short-term heart rate variability. Lung India 2012;29:254-8.

54. Madsen T, Christensen JH, Toft E, Schmidt EB. C-reactive protein is associated with heart rate variability. Ann Noninvasive Electrocardiol 2007;12:216-22.
55. Hotamisligil GS. Inflammation and metabolic disorders. Nature 2006;444:860-7.

56. Rea IM, Gibson DS, McGilligan V, McNerlan SE, Alexander HD, Ross OA. Age and age-related diseases: role of inflammation triggers and cytokines. Front Immunol 2018;9:586.

57. von Känel R, Nelesen RA, Mills PJ, Ziegler MG, Dimsdale JE. Relationship between heart rate variability, interleukin-6, and soluble tissue factor in healthy subjects. Brain Behav Immun 2008;22:461-8.

58. von Känel R, Carney RM, Zhao S, Whooley MA. Heart rate variability and biomarkers of systemic inflammation in patients with stable coronary heart disease: findings from the Heart and Soul Study. Clin Res Cardiol 2011;100:241-7.

59. Sajadieh A, Nielsen OW, Rasmussen V, Hein HO, Abedini S, Hansen JF. Increased heart rate and reduced heart-rate variability are associated with subclinical inflammation in middleaged and elderly subjects with no apparent heart disease. Eur Heart J 2004;25:363-70.

60. Thayer JF, Sternberg E. Beyond heart rate variability: vagal regulation of allostatic systems. Ann N Y Acad Sci 2006;1088: 361-72.

61. Viljoen M, Claassen N. Allostatic load and heart rate variability as health risk indicators. Afr Health Sci 2017;17:428-35.

62. Tracey KJ. The inflammatory reflex. Nature 2002;420:853-9.

63. Pavlov VA, Tracey KJ. The vagus nerve and the inflammatory reflex--linking immunity and metabolism. Nat Rev Endocrinol 2012;8:743-54.

64. Martin CR, Osadchiy V, Kalani A, Mayer EA. The brain-gutmicrobiome axis. Cell Mol Gastroenterol Hepatol 2018;6:13348.

65. Chung JW, Yan VC, Zhang H. Effect of acupuncture on heart rate variability: a systematic review. Evid Based Complement Alternat Med 2014;2014:819871.

66. Kim JD, Kim YJ, Soh KS. Change of heart rate variability by electro-acupuncture stimulus in rats. Korean J Meridian Acupoint 2007;24:185-91.

67. Yang Y, Litscher G, Sheng Z, Wang L. Heart rate variability during acupuncture treatment of lumbosacral pain. OBM Integr Complement Med 2020;5:13.

68. Wang G, Tian Y, Jia S, Zhou W, Zhang W. Acupuncture regulates the heart rate variability. J Acupunct Meridian Stud 2015; 8:94-8.

69. Moreira BR, Duque AP, Massolar CS, de Lima Pimentel R, Mediano MFF, Guimarães TCF, et al. Transcutaneous electrical stimulation of PC5 and PC6 acupoints modulates autonomic balance in heart transplant patients: a pilot study. J Acupunct Meridian Stud 2019;12:84-9.

70. Yoon DS, Hong SW, Lee YS. The effect of electroacupuncture at Sobu(HT8) on the EEG and HRV. Korean J Acupunct 2013;30: 305-18.

71. Lee BH, Chung JH, Son J, Heo JH, Park JH, Kim HY, et al. 
Influence of rotation number in the effect of PC6 on the heart rate: a pilot study. Korean J Acupunct 2013;30:88-96.

72. Shi X, Wang H, Wang L, Zhao Z, Litscher D, Tao J, et al. Can tongue acupuncture enhance body acupuncture? First results from heart rate variability and clinical scores in patients with depression. Evid Based Complement Alternat Med 2014;2014: 329746.

73. Sung KK. Effects of acupoint stimulation at the pericadium and liver meridian on heart rate variability. Korean J Acupunct 2015;32:130-5.

74. Kwak S, Choi WJ, Lee SG, Park KM. The effects of acupuncture stimulation and progressive relaxation therapy on examination stress of students - using heart rate variability and pupil size variability -. Korean J Meridian Acupoint 2004;21:161-76.

75. Ouyang H, Yin J, Wang Z, Pasricha PJ, Chen JD. Electroacupuncture accelerates gastric emptying in association with changes in vagal activity. Am J Physiol Gastrointest Liver Physiol 2002;282:G390-6.
76. Lee JH, Choi YJ, Shin BC, Koo ST. Differential effects of two individual acupuncture points (BL62, KI6) on heart rate variability in healthy volunteers : a randomized, single-blind, selfcontrolled trial. Korean J Acupunct 2010;27:85-97.

77. Li Z, Wang C, Mak AF, Chow DH. Effects of acupuncture on heart rate variability in normal subjects under fatigue and nonfatigue state. Eur J Appl Physiol 2005;94:633-40.

78. Pagani M, Lucini D, Mela GS, Langewitz W, Malliani A. Sympathetic overactivity in subjects complaining of unexplained fatigue. Clin Sci (Lond) 1994;87:655-61.

79. Quiroz-González S, Fossion R, López-Espinosa E, JiménezEstrada I. Analysis of the traditional characters of deqi and proposal for a biocircuit based on the general systems theory. Rev Int Acupunt 2021;15:73-85.

80. Quiroz-González S, Torres-Castillo S, López-Gómez RE, Jiménez Estrada I. Acupuncture points and their relationship with multireceptive fields of neurons. J Acupunct Meridian Stud 2017;10:81-9. 